Regular Article

\title{
Self-assembly of bacterial amyloid protein nanomaterials on solid surfaces
}

\author{
Tugce Onur ${ }^{\mathrm{a}, 1}$, Esra Yuca ${ }^{\mathrm{a}, \mathrm{b}, 1}$, Tolga Tarkan Olmez ${ }^{\mathrm{a}}$, Urartu Ozgur Safak Seker ${ }^{\mathrm{a}, *}$ \\ ${ }^{a}$ UNAM-Institute of Materials Science and Nanotechnology, Bilkent University, TR-06800 Ankara, Turkey \\ ${ }^{\mathrm{b}}$ Molecular Biology and Genetics Department, Yildiz Technical University, 34210 Istanbul, Turkey
}

\section{G R A P H I C A L A B S T R A C T}

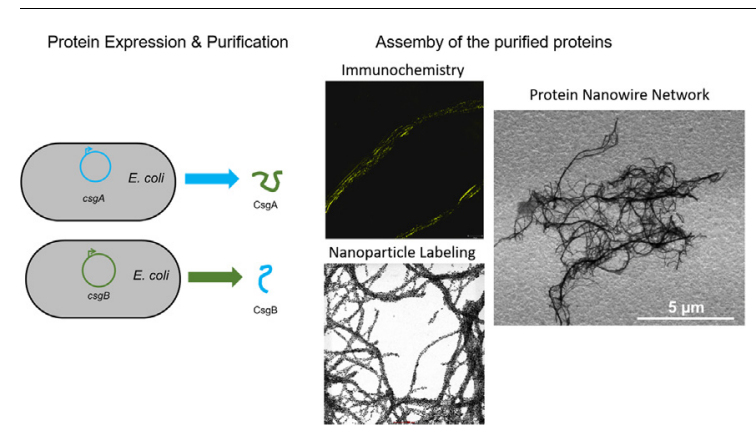

\section{A R T I C L E I N F O}

\section{Article history:}

Received 18 October 2017

Revised 4 March 2018

Accepted 6 March 2018

Available online 7 March 2018

\section{Keywords:}

Bacterial biofilm proteins

Curli

Amyloids

Nanomaterials

\begin{abstract}
A B S T R A C T
Hypothesis: Amyloid-forming biofilm proteins of Escherichia coli, namely CsgA and CsgB, can form selfassembled nanofibers on solid surfaces. These proteins can be programmed to form bio-nanomaterials for functional applications.

Experiments: In this study, the assembly of the CsgA and CsgB protein on solid surfaces was investigated in real time using a quartz crystal microbalance instrument with dissipation monitoring. The assembly kinetics of the CsgA and CsgB proteins in various settings on solid surfaces were investigated. Protein nanowires were investigated using electron microscopy.

Findings: CsgA protein polymers and CsgB-added CsgA polymers form densely packed biofilm on gold surfaces, whereas CsgB polymers and CsgA-added CsgB polymers form biofilms with high waterholding capacity according to the dissipation data. Electron microscopy images of nanofibers grown on gold surfaces showed that CsgA and CsgB polymers include thicker nanofibers compared to the nanofibers formed by CsgA-CsgB protein combinations. The resulting nano/microstructures were found to have strong fluorescence signals in aqueous environments and in chloroform while conserving the protein nanowire network.
\end{abstract}

(c) 2018 Elsevier Inc. All rights reserved.

\footnotetext{
* Corresponding author at: UNAM-Institute of Materials Science and Nanotechnology, National Nanotechnology Research Center, Bilkent University, TR-06800 Ankara, Turkey.

E-mail address: urartu@bilkent.edu.tr (U.O.S. Seker).

1 These authors contributed equally.
}

\section{Introduction}

Proteins form hierarchical material assemblies through selfassembly and molecular recognition [1-4]. They serve as scaffolds in tissue formation and biomineral deposition, or they feature hierarchical assemblies leading to complex material systems [5-9]. As monomeric material-forming units, proteins can 
be tuned in terms of structure and functionality through protein engineering $[10,11]$. In protein-based material systems, selfassembly is the driving force for the formation of complex materials [12].

Viral capsid proteins, cage proteins and amyloids can form self-assembled material systems [13,14]. Among these proteins, amyloids have the potential to form protein polymers with advanced mechanical and physical properties [14-16]. Amyloids can be grouped into pathological and functional amyloids as the major classes. Pathological amyloids are defined as the products of protein misfolding and cause many human diseases such as Alzheimer's, Parkinson's and Huntington's disease [17-19]. In amyloids, $\beta$-strands are elongated perpendicular to the fiber axes forming the final shape $[20,21]$. Hydrogen bonding between side chain groups affects the spacing of $\beta$ sheets [22]. Additionally, intermolecular interactions play a role in stabilization of the amyloid structure, which also gives rise to the mechanical properties of the amyloids [23,24]. The resulting amyloid structure is rigid and could resist protease treatment or environmental and chemical stress factors such as temperature and detergents $[25,26]$.

In addition to pathological amyloids, functional amyloids are also available in nature. Certain bacteria species, such as Salmonella typhimurium, Streptomyces coelicolor, Mycobacterium tuberculosis, and Escherichia coli, are capable of producing functional amyloids [27-31]. Functional amyloids of bacteria facilitate attachment of the biofilms onto hydrophilic or hydrophobic surfaces and invasion of host cells. Functional amyloids of E. coli are responsible for cellsurface attachment and cell-cell interactions in the biofilm matrix [32-34]. Synthesis and secretion of curli proteins are generated by two operons: $\operatorname{csg} B A C$ and $\operatorname{csg} D E F G$. The $\operatorname{csg} D E F G$ operon is responsible for transcription of proteins that regulate secretion of curli subunits. The major and minor subunits CsgA and CsgB, respectively, are transcribed by the $\operatorname{csg} B A C$ operon $[30,35]$. Following secretion, CsgA assembles onto the cell surface by the aid of CsgB, whereas CsgB anchors to the cell surface and serves as a nucleator for CsgA polymerization in vivo, as demonstrated in Fig. 1A [36,37]. Recently, CsgB was reported to be the critical protein for controlling the mechanical properties of E. coli biofilms [38]. However, both proteins could self-assemble in vitro (Fig. 1B). Pre-formed CsgA or CsgB fibers accelerate CsgA or CsgB polymer growth $[37,39,40]$.

In recent years, there has been increasing interest in the utilization of bacterial amyloids as functional material systems $[7,15,41,42]$. Engineered CsgA proteins were used as a cell surface display system for fluorescent proteins and nanomaterials, and recently, aromatic amino acid-inserted biofilm proteins were demonstrated to be innovative conductive interfaces [43]. Also, protein nanofibers of CsgA and CsgB can be utilized as an interface for material templating (for nanowire growth) [44], for ordered assembly of enzymes [45], for building underwater adhesives [46] and for creating conductive interfaces [43].

In this study, purified CsgA and CsgB proteins (secretion signals removed) were studied for their self-assembly kinetics on solid surfaces to understand the nature of the self-assembled protein nanowire network using a mass sensitive method coupled with dissipation monitoring. We observed that assembly behaviors of biofilm protein polymers on a gold surface are different depending on the type of the biofilm protein. Additionally, protein polymers were further analyzed for their functional properties in terms of structure and fluorescent emission capabilities. These finding provide a new approach to the assembly and functionality of biofilm proteins on solid surfaces. Some of the possible application areas of the final protein-based material systems are discussed in the conclusion part of this manuscript.

\section{Materials and methods}

\subsection{Cloning of $\operatorname{csg} A$ and $\operatorname{csg} B$ gene fragments}

Gene fragments of $\operatorname{csg} A$ and $\operatorname{csg} B$ were amplified from the $E$. coli MG1655 genome. GS linker sequences (composed of three repeats of GGGS amino acids) were added to the 3-prime end of gene fragments via PCR reactions. Flexible linkers were used to prevent protein-tag interactions and loss of protein function. Recombinant gene fragments were cloned into the $\mathrm{pET}-22 \mathrm{~b}(+)$ expression vector with the cut ligate method using HindIII and BamHI endonucleases (NEB). The pET-22b(+) expression vector enabled us to synthesize recombinant proteins with a 6-histidine-tag on $C$ terminals under control of the lacI promoter. Before the ligation step, the pelB leader sequence was removed from the original pET-22b(+) vector. We also designed hybrid proteins, which simultaneously had CsgA or CsgB and Venus Yellow fluorescent protein (YFP). To produce CsgA-YFP and CsgB-YFP fusion proteins, the Venus YFP-encoding DNA fragment was linked to the $3^{\prime}$ end of CsgA or CsgB. Recombinant plasmids were isolated with the QIAprep Spin Miniprep Kit. Plasmid constructs were sequence verified by Genewiz Inc. The primers used in this study are listed in Table S1. The DNA sequences of inserts and amino acid sequences of protein products are presented in Tables S2 and S3, respectively. Plasmid maps of the constructs are shown in supplementary Figs. S1 and S3. DNA sequencing results for the $\operatorname{csg} A$ and $\operatorname{csg} B$ genes and their alignments are presented in Figs. S2 and S4, respectively.

\subsection{Growth and purification of curli subunits}

Bacteria (E. coli BL21) that contained sequence verified recombinant plasmids were grown in starter culture at $37^{\circ} \mathrm{C}$ for $6-8 \mathrm{~h}$, and then the culture was diluted with a $1 / 5$ ratio to scale up and grow with overnight shaking. After overnight incubation, the cell culture was diluted again to a $1 / 5$ ratio to scale up and grow until the $\mathrm{OD}_{600}$ reached around 0.9 . Overexpression of curli proteins was started by induction of the synthetic lac operon on plasmids with isopropyl $\beta$-D-1-thiogalactopyranoside (IPTG, from Sigma-Aldrich, $\geq 99 \%$ (TLC)) ( $0.5 \mathrm{mM}$ final concentration). Induced cultures were incubated for two hours. Following the growth, cells were harvested with centrifugation at $4000 \mathrm{~g}$ for $20 \mathrm{~min}$, and the supernatant was removed. Purification was performed in denaturing conditions, and guanidine hydrochloride ( $\mathrm{GdnHCl}$, from Sigma-Aldrich, $\geq 99 \%$ ) was used as the denaturing agent. Cell pellets were suspended in phosphate buffered saline (PBS at $\mathrm{pH} 7.0$ ) that contained $6 \mathrm{M}$ $\mathrm{GdnHCl}$ and $10 \mathrm{mM}$ imidazole. Then, cobalt resin was added to the cell lysate and incubated for an hour at $+4{ }^{\circ} \mathrm{C}$. Resin was washed with the same buffer at $\mathrm{pH}$ 7.0. In addition, proteins were eluted with PBS buffer that contained $6 \mathrm{M} \mathrm{GdnHCl}$ and $150 \mathrm{mM}$ imidazole. A ThermoScientific ${ }^{\mathrm{TM}}$ Pierce ultrafiltration centrifugal device with a molecular-weight cutoff of $3 \mathrm{kDa}$ was used for buffer exchange of the purified protein samples. Eluted proteins were cleansed from $\mathrm{GdnHCl}$ and imidazole with PBS buffer. Then, $20 \mathrm{~mL}$ of PBS was used for $1 \mathrm{~mL}$ of protein eluent. The molecular weights of the recombinant proteins were calculated as CsgA $15.52 \mathrm{kDa}$, CsgB $13.34 \mathrm{kDa}$, CsgA-YFP $41.25 \mathrm{kDa}$ and CsgB-YFP $40 \mathrm{kDa}$ using the Swissprot Expasy tool. ${ }^{50}$ DNA sequencing results for the $\operatorname{csg} A-Y F P$ and $\operatorname{csg} B$ YFP genes and their alignments are presented in Figs. S5 and S6, respectively Finally, oligomers of CsgA and CsgB were separated from monomers with a $30-\mathrm{kDa}$ ultrafiltration centrifugal device, and the monomer samples were stored at $+4{ }^{\circ} \mathrm{C}$ (ThermoScientific ${ }^{\mathrm{TM}}$ Pierce). CsgA-YFP and CsgB-YFP fusions were not subjected to any centrifugal separations. Protein concentrations were determined with the bicinchoninic acid (BCA) assay according to the 
A

B

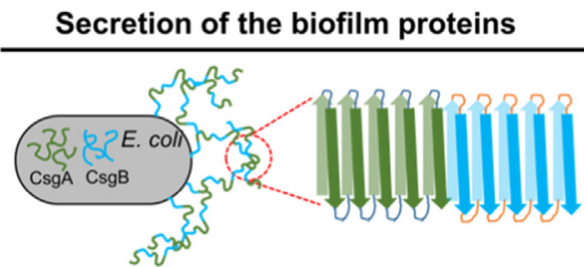

Protein Expression \& Purification

Assemby of the purified proteins

Curli fibers

Plasmids encoding CsgA and CsgB proteins
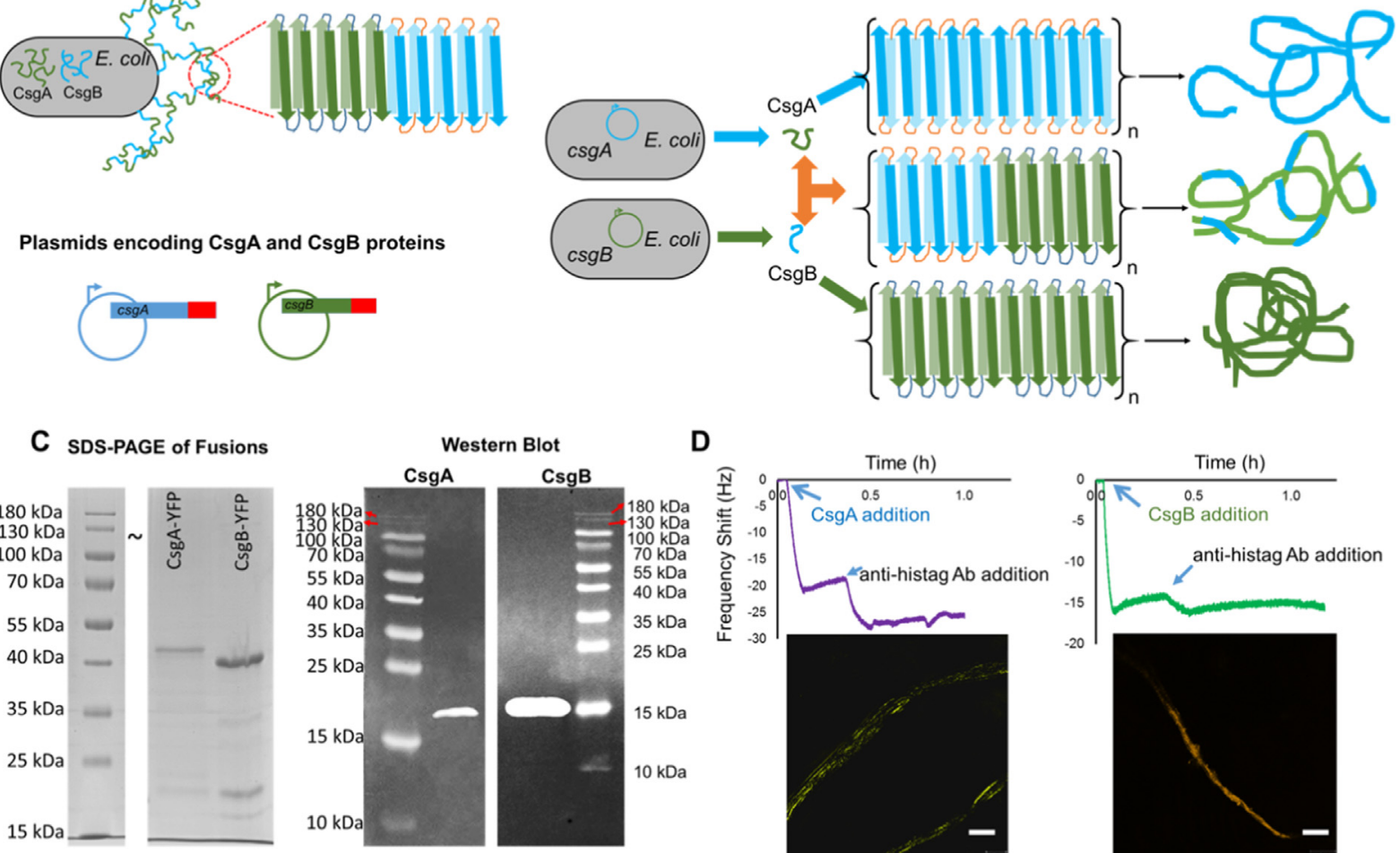

Western Blot

D
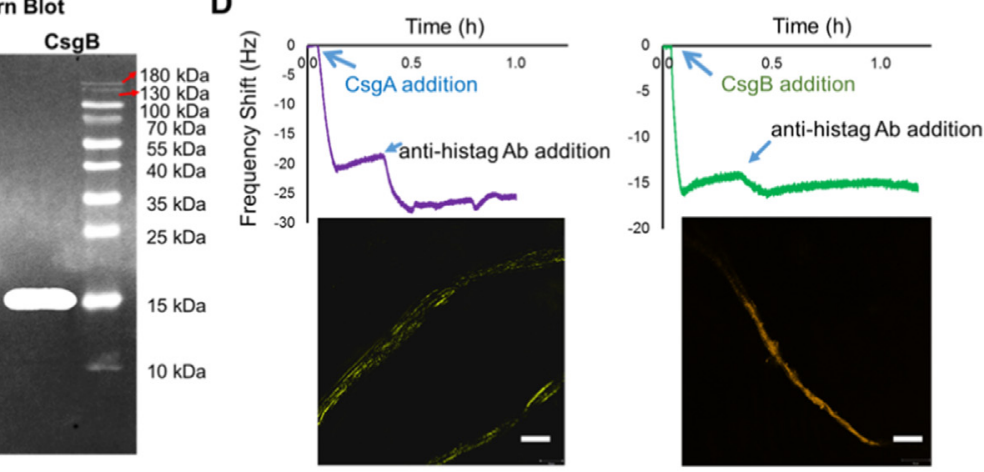

E

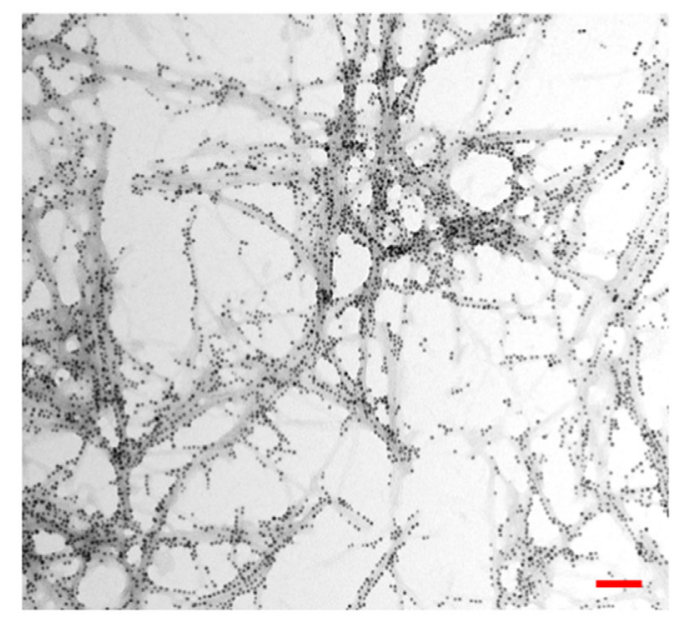

$\mathbf{F}$

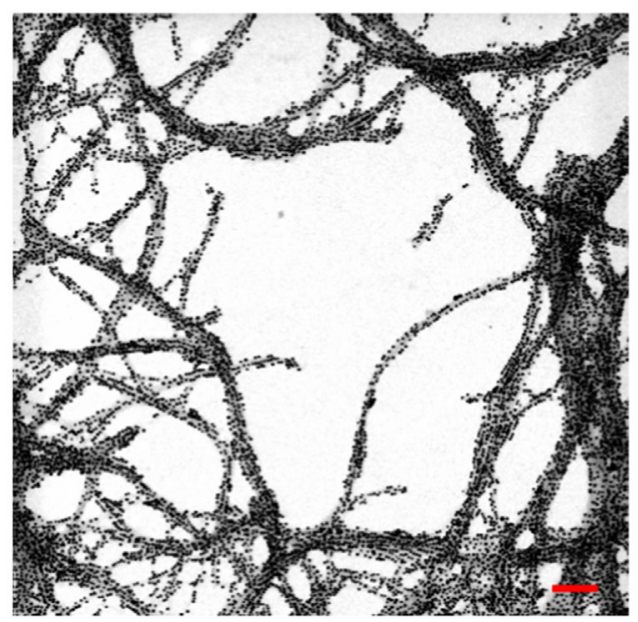

Fig. 1. (A) Secretion of the native curli biofilm proteins through the natural curli biosynthesis pathway. Once the operon is on, continuous secretion of CsgA and CsgB is enabled and the curli fiber network is formed. (B) Using the protein expression system, CsgA and CsgB proteins are expressed and purified with a soft linker and purification tag. Purified proteins are incubated in varying combinations: first CsgA-CsgA, CsgB-CsgA and finally CsgB-CsgB. All of the combinations resulted in hierarchical assemblies and complex networks. (C) SDS-PAGE of CsgA-YFP and CsgB-YFP fusion proteins and western blot analysis of the CsgA and CsgB proteins. (D) Validation of CsgA and CsgB proteins within the final fiber network. First, the CsgA and CsgB were added on the QCM sensor using a flow chamber, and later, antibodies and secondary fluorescent-labeled antibodies were added to visualize formed fibers (lines represent $50 \mu \mathrm{m}$ scale bar). (E) Transmission electron microscope images of histidine-tagged CsgA and (F) CsgB nanofibers are labeled with Ni-NTA-coated gold nanoparticles (lines represent $100 \mathrm{~nm}$ scale bar).

manufacturer's protocol (ThermoScientific Pierce). Approximately $900 \mu \mathrm{g}$ of his-tagged protein per liter of each bacterial culture producing CsgA and CsgB was obtained. Purified proteins were immediately placed at $+4{ }^{\circ} \mathrm{C}$ to delay any possible polymerization. The polymerization assay was started right after the purification and determination of protein concentrations.

\subsection{Western blot analysis}

Eluted proteins were treated with formic acid to break down the oligomers and polymers into soluble monomers of CsgA or CsgB proteins. Following formic acid treatment, samples were freezedried to remove formic acid as advised in previous studies [47]. 
Lyophilized samples were dissolved with Laemmli sample buffer and loaded onto SDS-PAGE gels (15\%). Afterwards, proteins were transferred to the polyvinylidene difluoride (PVDF, from Pierce) membrane using a trans-blot turbo system (Bio-Rad). Membrane blocking was performed at room temperature (RT) for an hour with $5 \%$ nonfat milk powder suspended in tris-buffered saline, $0.1 \%$ Tween 20 (TBS-T, from Sigma and VWR). Proteins were probed with 1:5000 dilutions of anti-his mouse primary antibody (Thermo Scientific Pierce) in TBS-T and 1:5000 dilutions of anti-mouse horseradish-peroxidase (HRP)-conjugated secondary antibody (Thermo Scientific Pierce) in TBS-T. Membranes were washed before and after incubation with secondary antibody. By the aid of enhanced chemiluminescence (ECL) substrate (Bio-Rad), the membrane was visualized with the Chemidoc MP gel imaging system (Bio-Rad).

\subsection{Polymerization assay}

Purified proteins were used for the polymerization assay in PBS. Polymerization of CsgA and CsgB proteins or their equimolar combinations was carried out in microcentrifuge tubes in PBS ( $\mathrm{pH} 7.0$ ). Right after the buffer exchange step, $96 \mu$ of purified proteins in PBS and thioflavin T (ThT) fluorescent dye were loaded onto 96well plates. The final concentration of ThT was determined as 20 $\mu \mathrm{M}$, and excitation and emission wavelengths were fixed at 438 and $495 \mathrm{~nm}$ (475-nm cutoff) to measure fluorescence intensities. Fluorescence intensities were measured every 10 min with $5 \mathrm{~s}$ of shaking before reading, and readings were recorded for fifteen hours using a Spectramax M5 plate reader. Fluorescence intensities were normalized as previously described in the literature [36]. The ThT assay results are presented in Fig. S7.

\subsection{Quartz crystal microbalance - dissipation (QCM-D) experiments}

Absorption and assembly of the purified proteins were monitored in real time using a quartz crystal microbalance Q-Sense E1. Prior to usage, sensor surfaces were placed in a mild piranha solution composed of DI water, ammonia (25\%) and hydrogen peroxide (30\%) (5:1:1 ratio) and heated for 5 min under a fume hood (CAUTION: Mild piranha solution is highly corrosive and should be handled with care). Following the piranha treatment to remove organics from the sensor surface, QCM-D sensors were incubated in DI water for $15 \mathrm{~min}$. Water droplets were removed from the sensor surfaces by blowing with nitrogen gas. Then, surfaces were treated under UV-ozone cleaner for 5 min to ensure surface cleanliness. The flow rate and temperature were fixed at $0.10 \mu \mathrm{l} / \mathrm{min}$ and $25^{\circ} \mathrm{C}$. The QCM-D sensor surface was washed with PBS until the frequency and dissipation signals reached equilibrium. After flowing the purified proteins in PBS buffer into the QCM-D flow cell, the sensor surface was washed again with PBS buffer to remove unbound or weakly bound soluble proteins. We carried out these experiments three times for each sample. Frequency and dissipation changes were recorded overnight by stopping the flow. The next day, freshly purified protein monomers were run through the system with diluted concentrations, and signal changes were also recorded overnight after PBS washing. At the end of the run, the system was washed with ultrapure water.

The addition of the CsgA proteins and growth of nanofibers was carried out as follows. Freshly purified $10 \mu \mathrm{M}$ CsgA was flowed into the QCM-D chamber and onto the gold-coated sensor surface. Following the polymerization of CsgA, on the next day, freshly purified CsgA was added to the system at diluted concentrations of $4 \mu \mathrm{M}, 5$ $\mu \mathrm{M}, 6 \mu \mathrm{M}$ and $7.5 \mu \mathrm{M}$. Similarly, $10 \mu \mathrm{M}$ freshly purified CsgB was flowed onto the sensor surface to form CsgB protein polymers. After overnight incubation, CsgB monomers were added to the system with diluted concentrations of $1.35 \mu \mathrm{M}, 1.8 \mu \mathrm{M}, 2.25 \mu \mathrm{M}, 2.7 \mu \mathrm{M}$ and $3.6 \mu \mathrm{M}$.

Freshly purified CsgB at diluted concentrations $(0.084 \mu \mathrm{M}$, $0.105 \mu \mathrm{M}, 0.126 \mu \mathrm{M}$ and $0.1575 \mu \mathrm{M}$ ) was deposited on the sensor surface that contained mature CsgA polymer. Then, CsgA monomers at diluted concentrations $(0.09 \mu \mathrm{M}, 0.12 \mu \mathrm{M}, 0.15 \mu \mathrm{M}, 0.18$ $\mu \mathrm{M}$ and $0.24 \mu \mathrm{M}$ ) were deposited on the sensor surface that was previously coated with CsgB polymer.

For the antibody binding assay, $2 \mu \mathrm{M}$ freshly purified CsgA was pumped onto the QCM-D sensor surface, and polymerization took place. Then, 1:5000 diluted anti-his mouse primary antibody (Thermo) in PBS was flowed through the system and then washed with PBS. When signals reached equilibrium, 1:5000 diluted fluorescent-labeled secondary antibodies (DyLight 550 Thermo) in PBS were added to the system. Next, the system was washed with PBS until both signals reached equilibrium. Sensors were observed with confocal laser scanning microscopy (CLSM, Zeiss LSM 510). The same procedure was performed with CsgB starting from $2 \mu \mathrm{M}$ freshly purified CsgB for polymerization.

\subsection{Fluorescence spectra recordings}

Fluoresce excitation and emission spectra of the purified proteins in PBS were measured with a Cary 100 Bio spectrophotometer. To carry out the measurements in chloroform solution, incubated CsgA proteins were centrifuged for half an hour to precipitate amyloid fibers. Then, the pellet was dissolved with chloroform and fluorescence excitation and emission spectra were measured. Each sample was investigated as three biological replicates.

\subsection{Imaging of the curli biomaterials}

The size and shape of curli fiber units formed in the QCM-D flow chamber on gold sensor surfaces were visualized by scanning electron microscopy (SEM) and fluorescence microscopy. For the SEM (FEI, USA) imaging studies, samples that were drop cast on silica or gold substrates were first air-dried and sputter coated with 5 $\mathrm{nm}$ of $\mathrm{Au} / \mathrm{Pd}$ alloy using a precision coating system (Gatan Inc, USA). Imaging was performed under high vacuum conditions with an electron beam energy of 5-15 KeV. Fluorescence microscopy studies were performed with an Axio Scope A1 (Zeiss, Germany) with excitation wavelengths of $546 \mathrm{~nm}$ for red, $455 \mathrm{~nm}$ for green and $365 \mathrm{~nm}$ for blue fluorescence and emission wavelengths of 575-640 nm, 500-550 nm and 420-470 nm, respectively. Fluorescence intensity exposure times were equal for the same area across different emissions.

\subsection{Gold nanoparticle assembly and transmission electron microscopy}

In vitro-formed biofilm fibers with histidine tags were labeled with nickel nitrilotriacetic acid- conjugated gold nanoparticles ( 5 $\mathrm{nm}$, Nanoprobes) with the protocol described in Chen et al. [48]. Briefly, the biofilm fibers were formed from freshly purified biofilm proteins in PBS (phosphate buffer saline) solution for two days at room temperature. The biofilm fibers were placed on TEM grids (formvar carbon-coated 200 mesh nickel grids (Electron Microscopy Sciences)). Then, $20 \mu$ d droplets containing biofilm fibers were placed on a piece of parafilm, and TEM grids were placed on droplets (face down) and incubated for five minutes. Then, TEM grids were washed with $30 \mu \mathrm{l}$ of $\mathrm{ddH}_{2} \mathrm{O}$ and selective binding buffer (1x PBS, $300 \mathrm{mM} \mathrm{NaCl}, 80 \mathrm{mM}$ imidazole, 0.2\% (v/v) tween20) for $5 \mathrm{~min}$. To label biofilm fibers with Ni-NTA-coated gold nanoparticles, TEM grids were placed on $90 \mu \mathrm{l}$ of $10 \mathrm{nM}$ NiNTAAuNP dissolved in selective binding buffer for $90 \mathrm{~min}$. During the labeling step, the droplet and TEM grid were covered with a petri 
dish to minimize evaporation of liquid. After the labeling step, the TEM grids were washed 5 times with $30 \mu$ l of selective binding buffer for 5 min each. The grids were washed 2 times with $30 \mu \mathrm{l}$ of $1 \mathrm{x}$ PBS and 2 times with $30 \mu \mathrm{l}$ of $\mathrm{ddH}_{2} \mathrm{O}$. Lastly, the TEM grids were stained with $20 \mu \mathrm{l}$ of $2 \%(\mathrm{w} / \mathrm{v})$ uranyl acetate for $15-30 \mathrm{sec}$. The TEM grids were air-dried. In all steps, excess liquid on TEM grids was wicked off with Whatman paper. The TEM grids were examined at $200 \mathrm{kV}$.

\section{Results and discussion}

\subsection{Production and validation of $C s g A$ and $C s g B$ proteins}

We designed CsgA and CsgB protein-coding constructs, and signaling sequences for both of the CsgA and CsgB genes were removed with an additional linker sequence coding region at the $3^{\prime}$ end of each gene. In the resulting protein, the GGGS soft linker [49] sequence along with the histidine purification tag was fused. Purified CsgA and CsgB proteins were verified using both SDSPAGE and western blotting as shown in Fig. 1C. Validation of the CsgA and CsgB proteins in the fiber structures was performed using both immunochemical methods and nanoparticle labeling. For immunochemical analysis of CsgA and CsgB proteins, antibody binding experiments were performed with fluorescent-labeled secondary antibodies by using QCM-D and imaged by confocal microscopy shown in Fig. 1D. In addition to the immunostaining of the polymerization in Fig. 1D, the amyloid formation was followed by the ThT assay (Supplementary Fig. S7). Additionally, gold nanoparticle labeling of the final curli nanofibers was carried out. His-tagged CsgA and CsgB proteins were purified, and nanofibers were formed after two days of incubation. Nanofibers displaying histidine tags were incubated with Ni-NTA 5-nm gold nanoparticles (AuNP), and after several washing steps, final assemblies were imaged with TEM. The TEM images in Fig. 1E and F show the presence of histidine on CsgA and CsgB nanofibers. In general, all of these validation steps were in agreement with each other.

To investigate the formation of characteristic sheet structures during functional amyloid formation, circular dichroism (CD) analysis of the proteins was performed. To carry out these analyses, purified proteins in PBS buffer at $\mathrm{pH} 7.0$ were incubated at RT for one day and fifteen days. Far-UV CD spectra of the aged samples were recorded, and secondary structure element ratios were analyzed quantitatively as described previously (Fig. S8) [50]. In all cases, that is, CsgA, CsgB and the CsgA-CsgB mix, sheet structures formed as the major secondary structure component as expected. This structure is the signature of amyloids. The $C D$ results revealed that incubation of CsgA for 15 days compared to the one day of incubation did not make any significant change in the helix, random coil or turn content, but it did change the sheet content. In the case of $\mathrm{CsgB}$, incubation of samples for 15 days did not make a significant change in secondary structure contents of the final fibrils. However, incubation for one day of CsgA and CsgB combination polymers compared to 15 days resulted in significant increases in the helix and sheet contents.

\subsection{Real-time monitoring of nanofiber assembly with QCM-D}

QCM-D offers real-time quantitative observation of materialprotein, protein-protein, and cell-cell interactions along with dissipated energy during surface interactions [51-53]. During proteinprotein and protein-surface interactions, conformational rearrangements of proteins can occur [54]. Such structural rearrangements could be monitored quantitatively by recording the frequency change and dissipation change as a function of the mass deposited. The dissipated energy is increasing if the surface-bound species are gel-like, which basically reflects the water-holding capacity of the proteins on the surface. A decrease in dissipation can be assumed to be a loss of water from the surface-bound structures and formation of a densely packed layer on the surface [5558]. Initial adsorption of CsgA and CsgB proteins to the goldcoated sensor surfaces of QCM-D and the assembly characteristics of freshly purified curli subunits were analyzed. The initial attachment of purified proteins was favored with the histidine tag, which is known to have affinity for the gold surface [59]. In our experimental setup, first, we tested polymerization of CsgA and CsgB proteins to investigate the interaction of the polymerized proteins with un-polymerized monomeric CsgA or CsgB proteins. CsgA and CsgB monomers were tested for their polymerization on the gold surface in Fig. 2. Initial surface attachments of monomers resulted in a sharp drop in frequency and sharp rise in the dissipation signal for all cases in Fig. 2. After pure CsgA or CsgB oligomers were deposited on the sensor surface, the flow was turned off and the growth of the protein polymers was monitored overnight. During polymerization of $\operatorname{Csg} A$, a decrease in the signal was observed after twelve hours. This change after twelve hours can be triggered by two possible reasons: first, the structural arrangement of CsgA on the surface, and second, the additional attachment of oligomers to CsgA polymers attached to the QCM-D gold sensor surface. Surfacetriggered structural changes were reported previously [60].

To monitor the interaction of the freshly purified protein monomers with polymers, $10 \mu \mathrm{M}$ CsgA and $10 \mu \mathrm{M}$ CsgB monomers were attached on QCM-D sensors in separate flow chambers. After $24 \mathrm{~h}$ of polymerization of the monomeric proteins, freshly purified CsgA or CsgB monomers were flowed on CsgA or CsgB polymers to monitor their interactions with the polymers. Purified protein monomers were added to the polymeric protein-covered sensors with increasing concentrations. Freshly purified CsgA proteins were sent to the sensor surface covered with CsgA polymer with an increasing concentration of the oligomeric CsgA (Fig. 3A). These data indicate that the CsgA polymers are likely to grow while a continuous resource of the CsgA oligomers and monomers is supplied. In Fig. 3B, freshly purified CsgB protein was added to polymerized CsgB proteins. Compared to the CsgA monomer-CsgA polymer, the addition of the CsgB monomer on the CsgB polymer caused a faster shift. This finding indicated that the CsgB-CsgB polymer interaction kinetics is faster than CsgA-CsgA polymer interactions. In Fig. 3C, freshly purified CsgB proteins were added to CsgA polymers, but a very low shift was observed as a result of weaker interaction kinetics between the polymers and monomer. In Fig. 3D, freshly purified CsgA was brought in contact with polymerized CsgB on the sensor surface. In both cases (fresh CsgA-CsgB polymer and CsgB-CsgA polymer), the dissipation did not change notably, indicating a densely packed protein layer formation on the surface. Mixtures of freshly purified proteins attached on surface-grown fibers for each of these four samples were kept on QCM-D sensor surfaces polymerized overnight.

In order to see if the structures in Fig. 3 were still forming additional attachment sites for CsgA and CsgB monomers after $24 \mathrm{~h}$ of incubation, the experiments presented in Fig. 4 were planned. Surface grown protein polymers (which are shown in Fig. 3) presented additional growth sites for the added monomers. Attachment of CsgA monomers to pre-formed CsgA polymers produces rigid structures in Figs. $4 \mathrm{~A}$ and $4 \mathrm{C}$ according to the very low shift in dissipation. In the case of CsgB (Fig. 4B) attachment on CsgB polymers and CsgA attachment on CsgB polymers, softer amyloid polymers were formed. This observation was supported by a dramatic increase in the dissipation change in Figs. 4B and 4D. In general, according to the dissipation data, assembled structures became more rigid after incubation for nearly two days.

CsgA and CsgB amyloid nanofibers grown on QCM-D gold sensors were investigated for their morphology under SEM. Images 
A

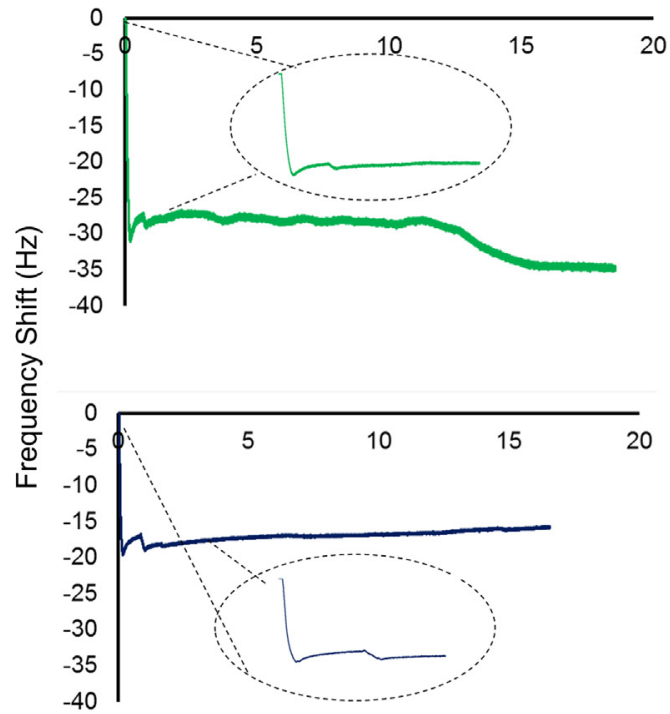

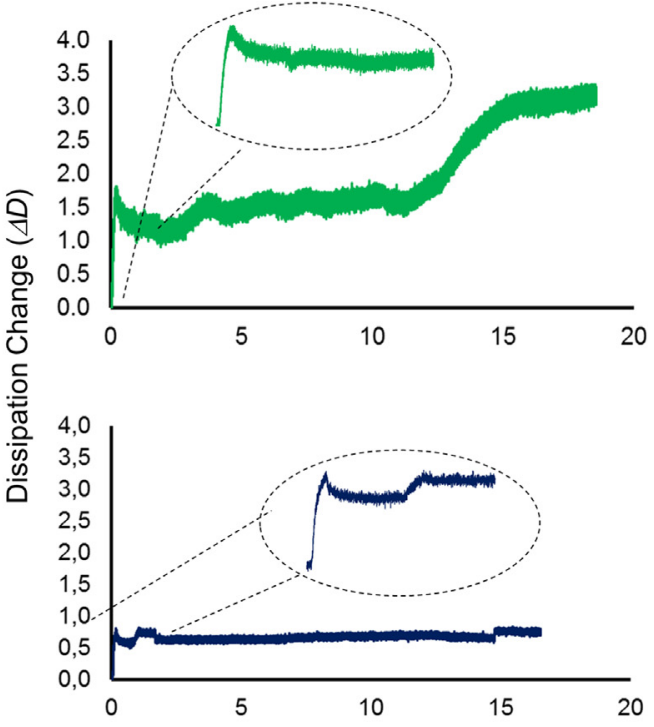

Time (h)

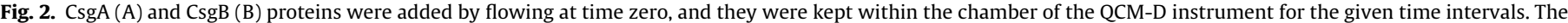

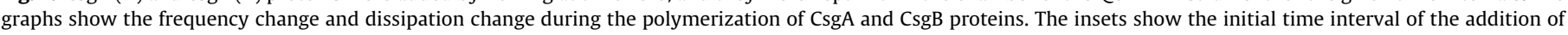
freshly purified protein onto the QCM-D sensor surface. The data points in circles show the addition of the monomers into the QCM-D chamber.

A

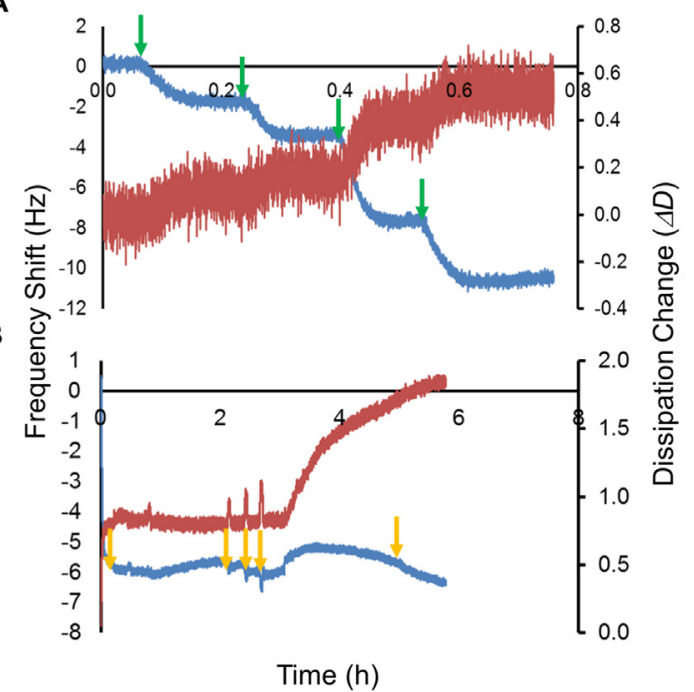

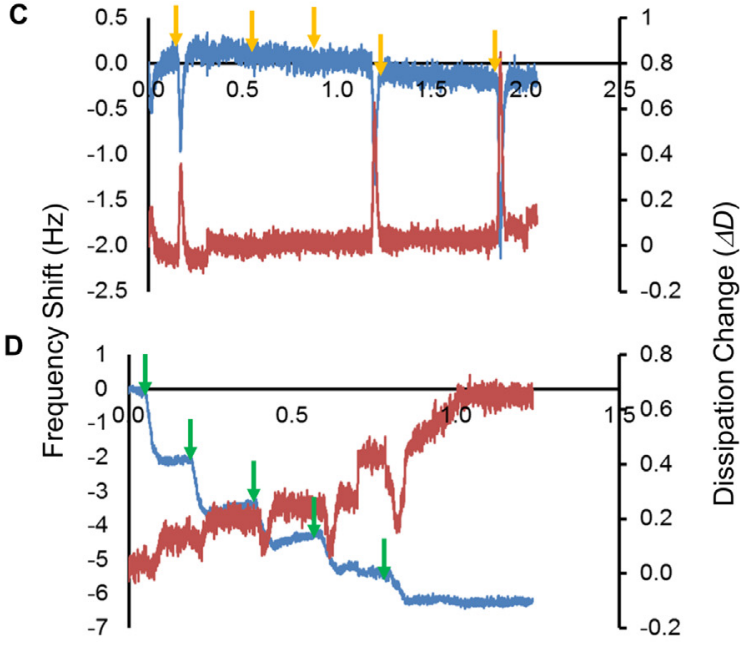

Time (h)

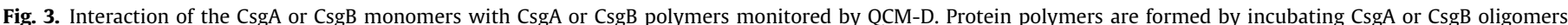

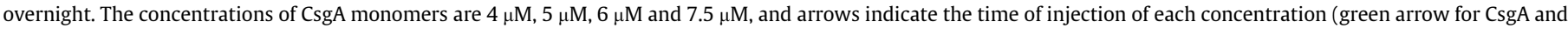

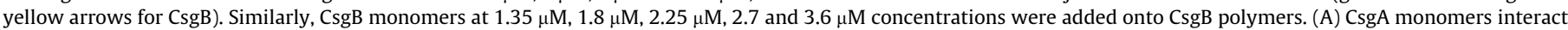

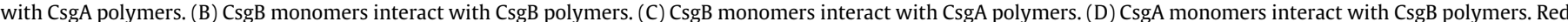

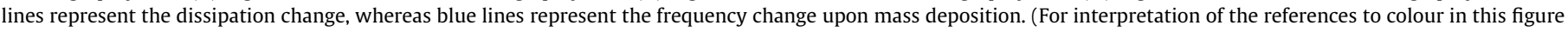
legend, the reader is referred to the web version of this article.)

of the final protein nanofibers formed by four different combinations are presented. Only CsgA nanofibers formed by addition of CsgA monomers on pre-polymerized CsgA are demonstrated in Fig. 5A, and only $\mathrm{CsgB}$ nanofibers formed by addition of monomeric CsgB on pre-polymerized CsgB nanofibers are in Fig. 5B. CsgB nanofibers are thicker than the CsgA nanofibers. However, nanofibers formed in both cases are thicker compared to the nanofibers formed as a result of CsgA added to CsgB polymers or CsgB added to CsgA polymers. These findings indicated that utilization of CsgA and $\mathrm{CsgB}$ proteins as a mixture results in different morphologies compared to only CsgA or only CsgB polymer cases. Utilization of this assembly route and controlling the concentrations or biochemical properties of each protein, nanofibers with useful features can be designed. Recently, a similar approach was applied to an in vivo system to control the mechanical properties of bacterial amyloid nanofibers [38]. 
A

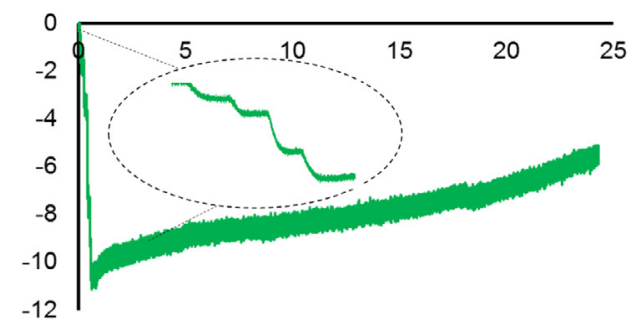

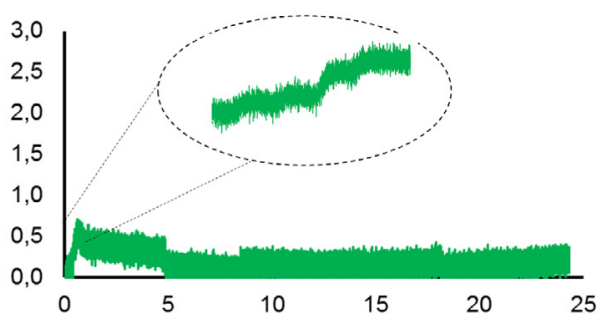

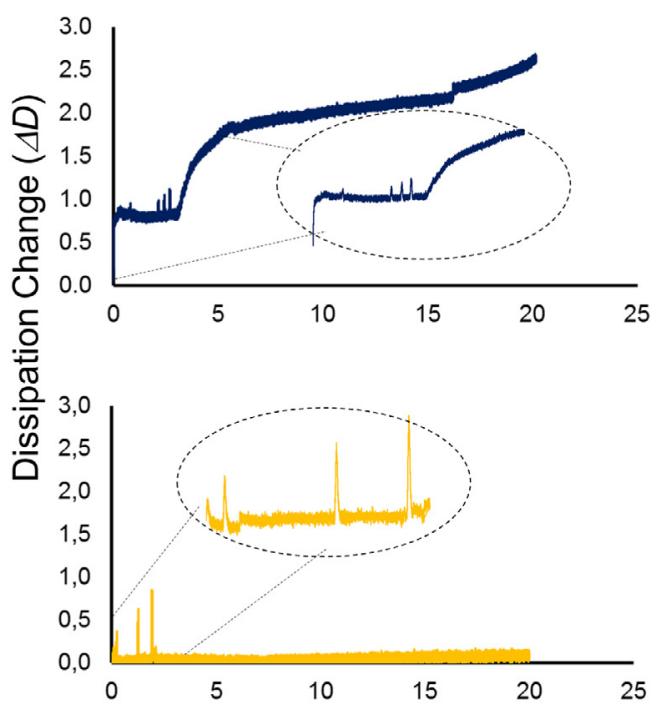

D

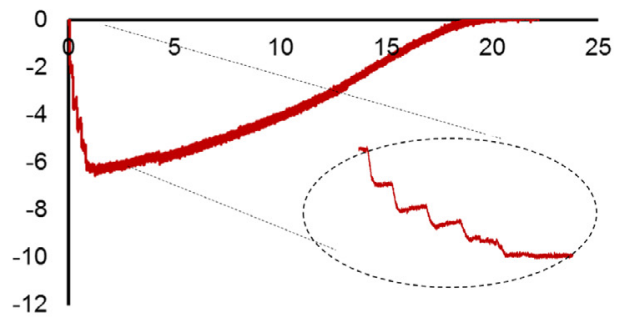

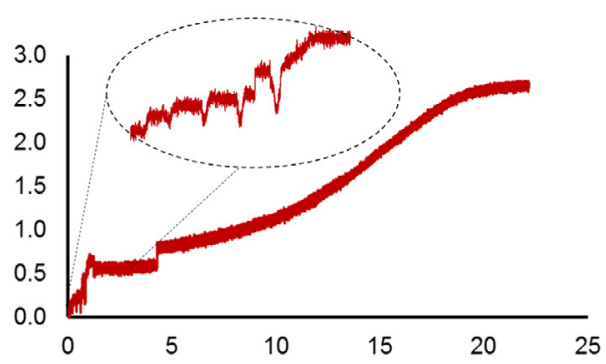

Time (h)

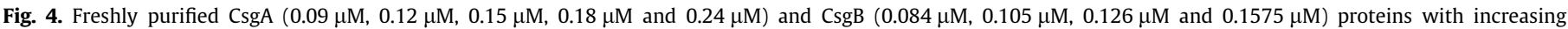

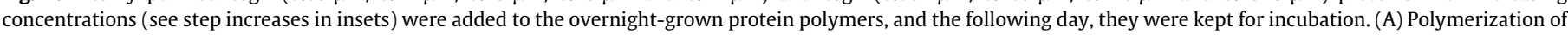

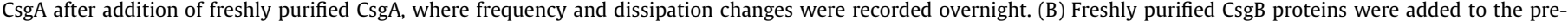

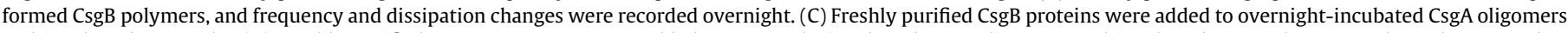

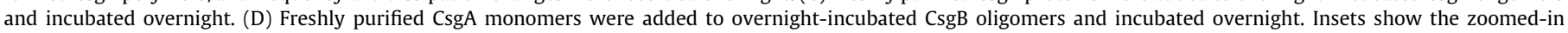
images of the initial attachment of proteins at varying concentrations on top of preformed protein polymers.

CsgA and CsgB proteins are capable of building self-assembled and organized structures. This opportunity can be used to design protein-based material assemblies to develop novel tools as biomaterials for controlled scaffold formation in tissue engineering. In tissue engineering applications, purified protein nanofibers of CsgA and CsgB proteins can be used as a new class of extracellular matrix mimicking protein nanofiber networks. To achieve this, some functional groups such as cell adhesion-promoting peptides can be fused to the CsgA and CsgB proteins.

\subsection{Analysis of the fluorescent properties of assembled biofilm protein nanofibers}

Intrinsic fluorescent properties of amyloids have been reported previously [61], but we investigated the progress of the intrinsic fluorescence of the CsgA and CsgB nanofibers for longer incubation periods. Additionally, the fluorescent properties of CsgB proteins and CsgA-CsgB mixtures have not been reported. Excitation and emission spectra of purified proteins in PBS buffer were determined, and measurements were performed. The results from incubating samples for nine days are shown in Fig. 6, and other samples (eighteen hours and twenty-three days of incubation) are shown in supplementary Figs. S9 and S10. CsgA samples were excited at 338, 375 and $550 \mathrm{~nm}$, while CsgB samples were excited at 339, 413 and $550 \mathrm{~nm}$. We excited protein polymers at varying wavelengths and collected the emission spectrum for each wavelength. Moreover, fluorescence intensity was determined for mixed solutions that were prepared by mixing CsgA and CsgB in equimolar concentrations. The highest fluorescence emission was detected at $340 \mathrm{~nm}$ excitation. As shown in Fig. 6, samples were visualized under a fluorescent microscope with proper filters. In order to understand if the emission spectrum is enabled through the buffer-protein poly- 

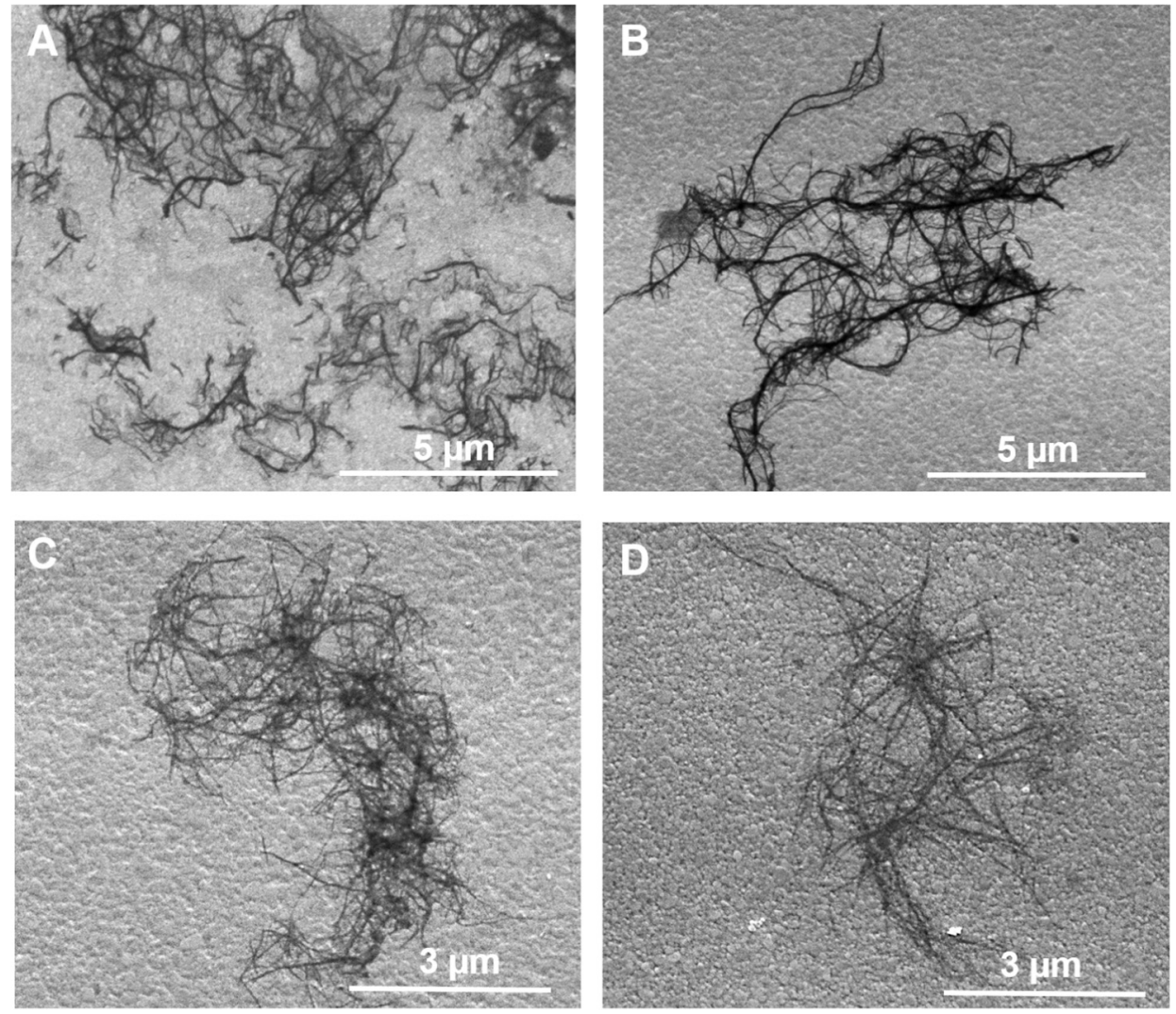

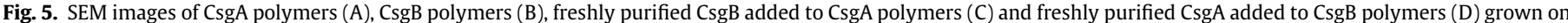
gold-coated QCM-D sensors. In all cases, the same monomer concentrations were used.

A

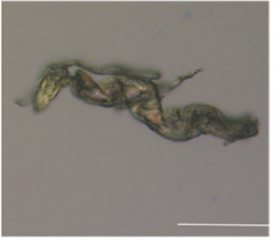

B

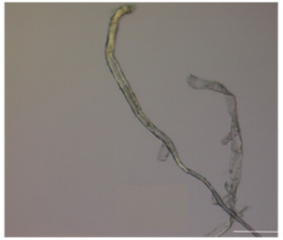

C

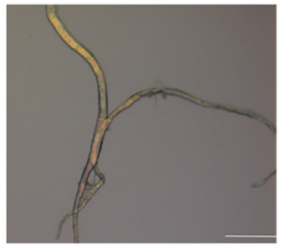

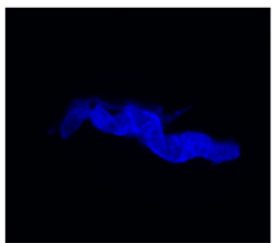
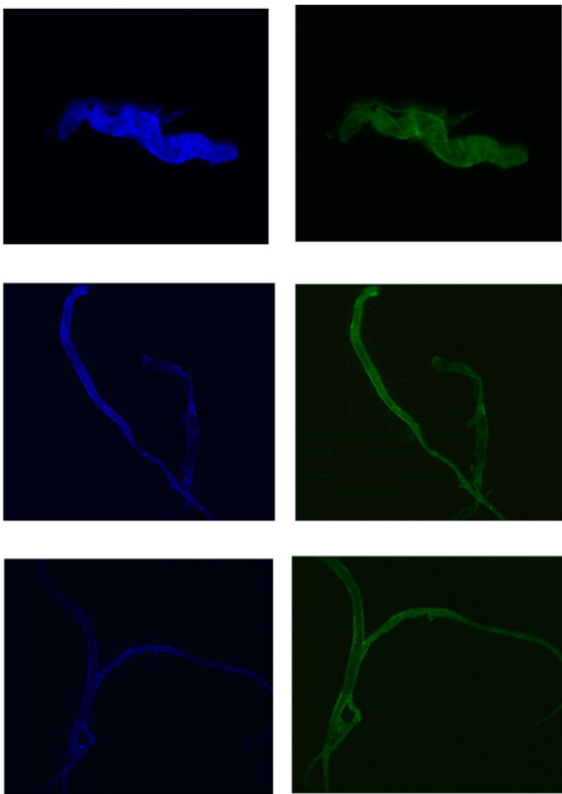
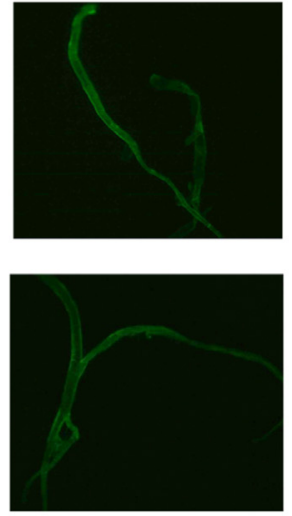
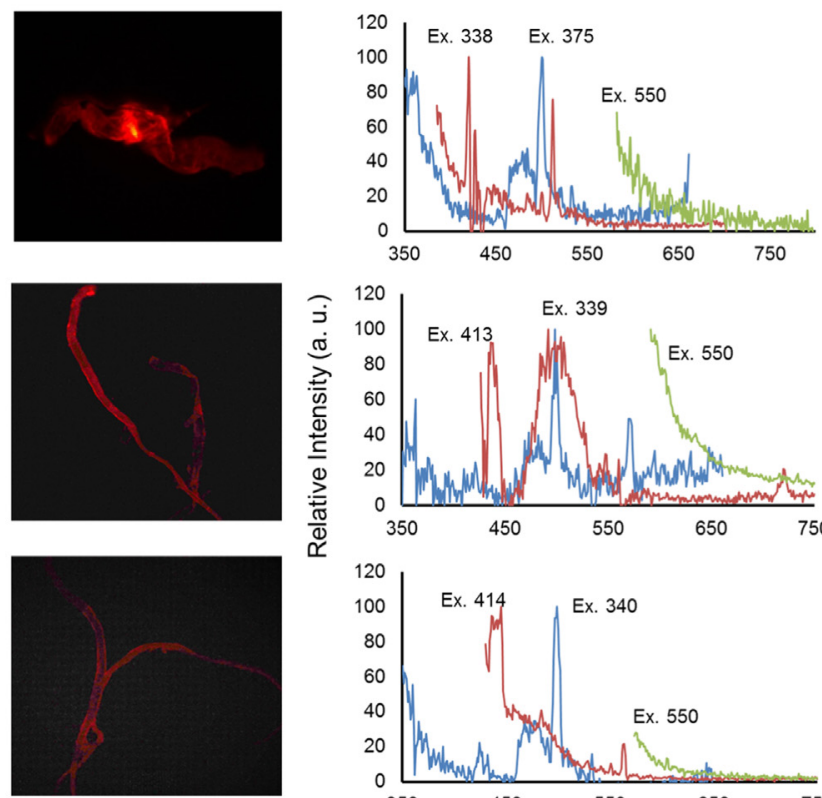
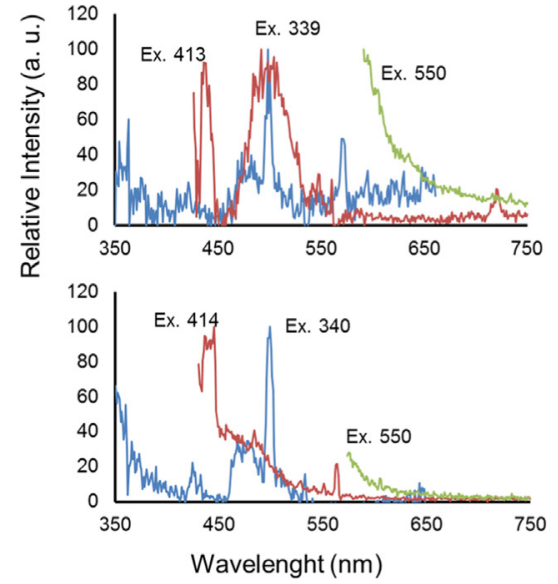

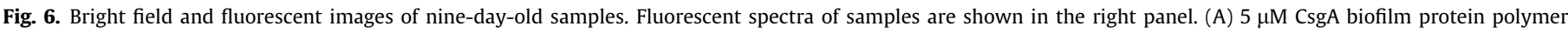

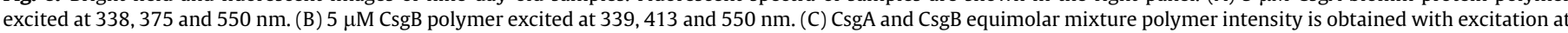
$340,414 \mathrm{~nm}$ and $550 \mathrm{~nm}$. Lines represent $100 \mu \mathrm{M}$ scale bar, and each of the fluorescent spectra belongs to the protein polymer on the same row.

mer interaction, we suspended the protein polymers in the organic solvent chloroform and measured the emission spectra for the CsgA polymer in Fig. 7. We observed broad emission spectra for
CsgA in chloroform, which is not very typical for proteins. The electron microscopy investigation of the CsgA suspended in chloroform revealed an undamaged fibril network of the self-assembled pro- 
A

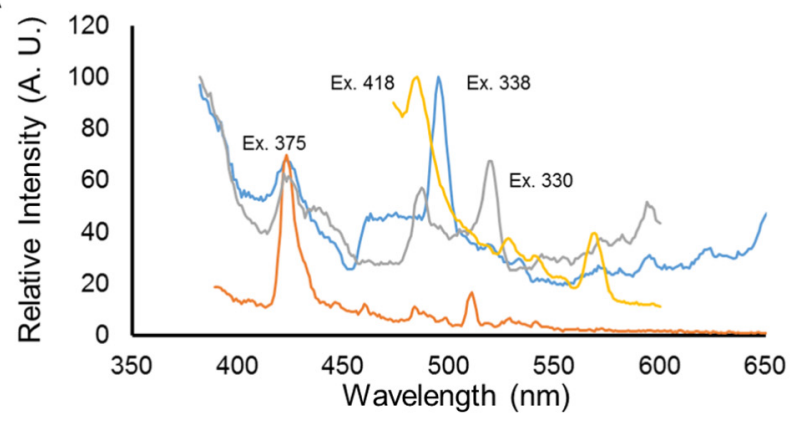

B

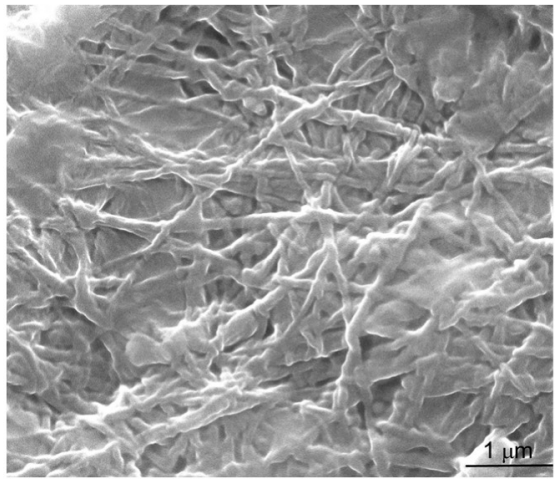

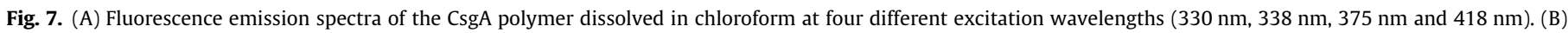
SEM image of the CsgA polymer suspended and incubated in chloroform, with protein fiber networks intact.

tein structure. In general, biofilm protein polymers are optically active, can be excited at varying wavelengths, and corresponding emissions can be gathered. Similar to their assembly characteristics, the route of assembly results in different optical properties in polymerized biofilm proteins.

\section{Conclusions}

Here, we demonstrated the formation of self-assembled materials of pure biofilm proteins of $E$. coli. Highly ordered protein materials of CsgA and CsgB proteins can form large polymeric structures with defined fluorescent emissions. Fluorescent biofilm protein materials can be used for a number of applications. The possibility of utilization of amyloids as a part of light-emitting diodes was studied previously [62]. It has been demonstrated that the conjugation of amyloid fibrils to LEDs yields a 10 -fold increase in external quantum efficiency. Solin et al. and Wei et al. have discussed these applications in detail. Similarly, self-fluorescent curli amyloids can be used in optoelectronic device settings in combination with fluorescent nanoparticles for templating them on solid surfaces and for facilitating a FRET-based energy transfer by playing a role as a donor/acceptor. Curli fibers have a distinct intrinsic fluorescent property that may enable them to be used within such FRET-based applications for bio-LEDs. Curli nanowires can also be programmed to form quantum dot-like structures for nanotechnology applications due to their morphology-dependent optical properties. Biomolecule-based materials are of great importance in biomedical applications as they have tunable mechanical properties. In the literature, there are lots of studies dealing with the fabrication of protein/peptide (elastin, resilin, silk, callgen, capsid, amyloids)-based smart biomaterials (Bracalello, Zhang, Woolfson). Among them, amyloidogenic peptides and proteins have great potential for use in various fields such as tissue regeneration, three-dimensional scaffold formation, cage formation for controlled drug release, coating materials, conductive wires, biosensors and bioswitches (Cherny, Chung, Knowles). Natural amyloids with adhesive properties function by attaching to various surfaces. Amyloidogenic proteins have been used in biofunctionalization of surfaces in different studies. Hydrophobins have been used with the help of their amphipathic nature to modify the chemical properties of surfaces to improve biocompatibility and have been shown to serve as scaffolds for tissue engineering (Gebbing et al.). Amphiphilic peptides that are artificially produced and can undergo ordered assembly in a liquid medium may support cell proliferation and have great potential in tissue engineering (Zhao). Amyloid peptides are designed to increase the carbon dioxide capture capacity and gene transfer efficiency (Li). In addition, a protein film was formed from the hierarchically assembled amy- loid fibers (Knowles). Recombinant production of protein/peptide ingredients allows biomaterials to be tuned and modified genetically. By covering all of this background, biofilm proteins can be engineered to extend their spectrum of applications using protein engineering approaches. They are capable of forming selfassembled hierarchical structures that can be tuned with different functionalities by the fusion of bioactive peptides or enzymes or other functional proteins using recombinant DNA techniques. These polymers are not only soluble and active in an aqueous environment but in solvent conditions as well. Combining the selfassembly capability of biofilm proteins with additional functionalities, they can be programmed for nanomaterial synthesis and assembly. With the addition of enzymes through protein engineering, they can form biocatalytic ordered networks, and with the fusion of signaling peptides, they can be used for regenerative medicine. However, there is still a gap in understanding how to design a well-regulated system to control nanofiber networks with desired physical and biological characteristics. This challenge can be achieved with the help of fundamental research combining nanotechnology and genetic engineering approaches.

\section{Acknowledgments}

We are thankful to TÜBITAK for financial support (project number 114M163). UOSS acknowledges the TÜBA-GEBIP Award. UOSS also thanks the Science Academy Distinguished Young Scientist Award (BAGEP) for financial support. TTO is thankful for the TÜBITAK-BIDEEB PhD fellowship. We also thank Prof. Dr. Hilmi Volkan Demir for allowing us to use the QCM-D instrument in his laboratory.

\section{Appendix A. Supplementary material}

Supplementary data associated with this article can be found, in the online version, at https://doi.org/10.1016/j.jcis.2018.03.016.

\section{References}

[1] P.C. Jordan, D.P. Patterson, K.N. Saboda, E.J. Edwards, H.M. Miettinen, G. Basu, M.C. Thielges, T. Douglas, Self-assembling biomolecular catalysts for hydrogen production, Nat. Chem. 8 (2) (2016) 179-185.

[2] Y.-T. Lai, E. Reading, G.L. Hura, K.-L. Tsai, A. Laganowsky, F.J. Asturias, J.A. Tainer, C.V. Robinson, T.O. Yeates, Structure of a designed protein cage that self-assembles into a highly porous cube, Nat. Chem. 6 (12) (2014) 1065-1071.

[3] M. Sutter, D. Boehringer, S. Gutmann, S. Guenther, D. Prangishvili, M.J. Loessner, K.O. Stetter, E. Weber-Ban, N. Ban, Structural basis of enzyme encapsulation into a bacterial nanocompartment, Nat. Struct. Mol. Biol. 15 (9) (2008) 939-947.

[4] C.A. Kerfeld, M.R. Sawaya, S. Tanaka, C.V. Nguyen, M. Phillips, M. Beeby, T.O. Yeates, Protein structures forming the shell of primitive bacterial organelles, Science 309 (5736) (2005) 936-938. 
[5] K.M. Woo, J.-H. Jun, V.J. Chen, J. Seo, J.-H. Baek, H.-M. Ryoo, G.-S. Kim, M.J. Somerman, P.X. Ma, Nano-fibrous scaffolding promotes osteoblast differentiation and biomineralization, Biomaterials 28 (2) (2007) 335-343.

[6] S.G. Zhang, Emerging biological materials through molecular self-assembly, Biotechnol. Adv, 20 (5-6) (2002) 321-339.

[7] T.P.J. Knowles, M.J. Buehler, Nanomechanics of functional and pathological amyloid materials, Nat. Nanotechnol. 6 (8) (2011) 469-479.

[8] K. Rajagopal, J.P. Schneider, Self-assembling peptides and proteins for nanotechnological applications, Curr. Opin. Struct. Biol. 14 (4) (2004) 480-486.

[9] S.G. Zhang, Fabrication of novel biomaterials through molecular self-assembly, Nat. Biotechnol. 21 (10) (2003) 1171-1178.

[10] N.K. Dutta, N.R. Choudhury, M.Y. Truong, M. Kim, C.M. Elvin, A.J. Hill, Physical approaches for fabrication of organized nanostructure of resilin-mimetic elastic protein rec1-resilin, Biomaterials 30 (28) (2009) 4868-4876.

[11] R.M. Kramer, W.J. Crookes-Goodson, R.R. Naik, The self-organizing properties of squid reflectin protein, Nat. Mater. 6 (7) (2007) 533-538.

[12] R. Jansson, C.M. Courtin, M. Sandgren, M. Hedhammar, Rational design of spider silk materials genetically fused with an enzyme, Adv. Funct. Mater. 25 (33) (2015) 5343-5352.

[13] S. Cao, M. Saha, W. Zhao, P.J. Jardine, W. Zhang, S. Grimes, M.C. Morais, Insights into the structure and assembly of the bacteriophage phi 29 double-stranded DNA packaging motor, J. Virol. 88 (8) (2014) 3986-3996.

[14] A. Valbuena, M.G. Mateu, Quantification and modification of the equilibrium dynamics and mechanics of a viral capsid lattice self-assembled as a protein nanocoating, Nanoscale 7 (36) (2015) 14953-14964.

[15] D. Li, E.M. Jones, M.R. Sawaya, H. Furukawa, F. Luo, M. Ivanova, S.A. Sievers, W. Wang, O.M. Yaghi, C. Liu, D.S. Eisenberg, Structure-based design of functional amyloid materials, J. Am. Chem. Soc. 136 (52) (2014) 18044-18051.

[16] S. Bolisetty, R. Mezzenga, Amyloid-carbon hybrid membranes for universal water purification, Nat. Nanotechnol. 11 (4) (2016) 365.

[17] M.S. Goldberg, P.T. Lansbury, Is there a cause-and-effect relationship between alpha-synuclein fibrillization and Parkinson's disease?, Nat Cell Biol. 2 (7) (2000) E115-E119.

[18] A. Kaushik, R.D. Jayant, S. Tiwari, A. Vashist, M. Nair, Nano-biosensors to detect beta-amyloid for Alzheimer's disease management, Biosens. Bioelectron. 80 (2016) 273-287.

[19] G. Hoffner, P. Djian, Polyglutamine aggregation in Huntington disease: does structure determine toxicity?, Mol Neurobiol. 52 (3) (2015) 1297-1314.

[20] J.W. Kelly, Alternative conformations of amyloidogenic proteins govern their behavior, Curr. Opin. Struct. Biol. 6 (1) (1996) 11-17.

[21] R. Nelson, M.R. Sawaya, M. Balbirnie, A.O. Madsen, C. Riekel, R. Grothe, D. Eisenberg, Structure of the cross-beta spine of amyloid-like fibrils, Nature 435 (7043) (2005) 773-778.

[22] M.T. Krejchi, S.J. Cooper, Y. Deguchi, E.D.T. Atkins, M.J. Fournier, T.L. Mason, D. A. Tirrell, Crystal structures of chain-folded antiparallel beta-sheet assemblies from sequence-designed periodic polypeptides, Macromolecules 30 (17) (1997) 5012-5024.

[23] C. Bortolini, N.C. Jones, S.V. Hoffmann, C. Wang, F. Besenbacher, M. Dong, Mechanical properties of amyloid-like fibrils defined by secondary structures, Nanoscale 7 (17) (2015) 7745-7752.

[24] T. Steckmann, Z. Awan, B.S. Gerstman, P.P. Chapagain, Kinetics of peptide secondary structure conversion during amyloid beta-protein fibrillogenesis, J. Theor. Biol. 301 (2012) 95-102.

[25] I. Kheterpal, S. Zhou, K.D. Cook, R. Wetzel, A beta amyloid fibrils possess a core structure highly resistant to hydrogen exchange, Proc. Natl. Acad. Sci. USA 97 (25) (2000) 13597-13601.

[26] A.K. Syed, B.R. Boles, Fold modulating function: bacterial toxins to functional annyloids, Front. Microbiol. 5 (2014) 9.

[27] C.L.L. Pham, A.H. Kwan, M. Sunde, Functional amyloid: widespread in Nature, diverse in purpose, in: S. Perrett (Ed.), Amyloids in Health and Disease, Portland Press Ltd, London, 2014, pp. 207-219.

[28] D. Claessen, R. Rink, W. de Jong, J. Siebring, P. de Vreugd, F.G.H. Boersma, L. Dijkhuizen, H.A.B. Wosten, A novel class of secreted hydrophobic proteins is involved in aerial hyphae formation in Streptomyces coelicolor by forming amyloid-like fibrils, Genes Dev. 17 (14) (2003) 1714-1726.

[29] C.J. Alteri, J. Xicohtencatl-Cortes, S. Hess, G. Caballero-Olin, J.A. Giron, R.L. Friedman, Mycobacterium tuberculosis produces pili during human infection, Proc. Natl. Acad. Sci. USA 104 (12) (2007) 5145-5150.

[30] M.R. Chapman, L.S. Robinson, J.S. Pinkner, R. Roth, J. Heuser, M. Hammar, S. Normark, S.J. Hultgren, Role of Escherichia coli curli operons in directing amyloid fiber formation, Science 295 (5556) (2002) 851-855.

[31] U. Romling, Z. Bian, M. Hammar, W.D. Sierralta, S. Normark, Curli fibers are highly conserved between Salmonella typhimurium and Escherichia coli with respect to operon structure and regulation, J. Bacteriol. 180 (3) (1998) 722731.

[32] M.Q. Carter, J.W. Louie, D. Feng, W. Zhong, M.T. Brandl, Curli fimbriae are conditionally required in Escherichia coli 0157:H7 for initial attachment and biofilm formation, Food Microbiol. 57 (2016) 81-89.

[33] I. Cherny, L. Rockah, O. Levy-Nissenbau, U. Gophna, E.Z. Ron, E. Gazit, The formation of Escherichia coli curli amyloid fibrils is mediated by prion-like peptide repeats, J. Mol. Biol. 352 (2) (2005) 245-252.

[34] J. Patel, M. Sharma, S. Ravishakar, Effect of curli expression and hydrophobicity of Escherichia coli 0157:H7 on attachment to fresh produce surfaces, J. Appl. Microbiol. 110 (3) (2011) 737-745.

[35] Z. Bian, S. Normark, Nucleator function of CsgB for the assembly of adhesive surface organelles in Escherichia coli, Embo J. 16 (19) (1997) 5827-5836.
[36] Q. Shu, S.L. Crick, J.S. Pinkner, B. Ford, S.J. Hultgren, C. Frieden, The E. coli CsgB nucleator of curli assembles to beta-sheet oligomers that alter the CsgA fibrillization mechanism, Proc. Natl. Acad. Sci. USA 109 (17) (2012) $6502-$ 6507.

[37] N.D. Hammer, B.A. McGuffie, Y. Zhou, M.P. Badtke, A.A. Reinke, K. Brannstrom, J.E. Gestwicki, A. Olofsson, F. Almqvist, M.R. Chapman, The C-terminal repeating units of CsgB direct bacterial functional amyloid nucleation, J. Mol. Biol. 422 (3) (2012) 376-389.

[38] M.T. Abdelwahab, E. Kalyoncu, T. Onur, M.Z. Baykara, U.O.S. Seker, Geneticallytunable mechanical properties of bacterial functional amyloid nanofibers, Langmuir 33 (17) (2017) 4337-4345.

[39] M. Sunde, L.C. Serpell, M. Bartlam, P.E. Fraser, M.B. Pepys, C.C.F. Blake, Common core structure of amyloid fibrils by synchrotron X-ray diffraction, J. Mol. Biol. 273 (3) (1997) 729-739.

[40] X. Wang, D.R. Smith, J.W. Jones, M.R. Chapman, In vitro polymerization of a functional Escherichia coli amyloid protein, J. Biol. Chem. 282 (6) (2007) 37133719 .

[41] T.P.J. Knowles, T.W. Oppenheim, A.K. Buell, D.Y. Chirgadze, M.E. Welland Nanostructured films from hierarchical self-assembly of amyloidogenic proteins, Nat. Nanotechnol. 5 (3) (2010) 204-207.

[42] X. Wang, Y. Li, C. Zhong, Amyloid-directed assembly of nanostructures and functional devices for bionanoelectronics, J. Mater. Chem. B 3 (25) (2015) 4953-4958.

[43] E. Kalyoncu, R.E. Ahan, T.T. Olmez, U.O.S. Seker, Genetically encoded conductive protein nanofibers secreted by engineered cells, RSC Adv. 7 (52) (2017) 32543-32551.

[44] U.O.S. Seker, A.Y. Chen, R.J. Citorik, T.K. Lu, Synthetic biogenesis of bacterial amyloid nanomaterials with tunable inorganic-organic interfaces and electrical conductivity, ACS Synth. Biol. 6 (2) (2017) 266-275.

[45] Z. Botyanszki, P.K.R. Tay, P.Q. Nguyen, M.G. Nussbaumer, N.S. Joshi, Engineered catalytic biofilms: site-specific enzyme immobilization onto E-coli curli nanofibers, Biotechnol. Bioeng. 112 (10) (2015) 2016-2024.

[46] C. Zhong, T. Gurry, A.A. Cheng, J. Downey, Z. Deng, C.M. Stultz, T.K. Lu, Strong underwater adhesives made by self-assembling multi-protein nanofibres, Nat. Nanotechnol. 9 (10) (2014) 858-866.

[47] M. Hammar, Z. Bian, S. Normark, Nucleator-dependent intercellular assembly of adhesive curli organelles in Escherichia coli, P Natl. Acad. Sci. USA 93 (13) (1996) 6562-6566.

[48] A.Y. Chen, Z.T. Deng, A.N. Billings, U.O.S. Seker, M.Y. Lu, R.J. Citorik, B. Zakeri, T. K. Lu, Synthesis and patterning of tunable multiscale materials with engineered cells, Nat. Mater. 13 (5) (2014) 515-523.

[49] W. Wriggers, S. Chakravarty, P.A. Jennings, Control of protein functional dynamics by peptide linkers, Biopolymers 80 (6) (2005) 736-746.

[50] A. Micsonai, F. Wien, L. Kernya, Y.H. Lee, Y. Goto, M. Refregiers, J. Kardos, Accurate secondary structure prediction and fold recognition for circular dichroism spectroscopy, PNAS 112 (24) (2015) E3095-E3103.

[51] E. Yuca, A.Y. Karatas, U.O.S. Seker, M. Gungormus, G. Dinler-Doganay, M. Sarikaya, C. Tamerler, In vitro labeling of hydroxyapatite minerals by an engineered protein, Biotechnol. Bioeng. 108 (5) (2011) 1021-1030.

[52] C. Modin, A.L. Stranne, M. Foss, M. Duch, J. Justesen, J. Chevallier, L.K. Andersen, A.G. Hemmersam, F.S. Pedersen, F. Besenbacher, QCM-D studies of attachment and differential spreading of pre-osteoblastic cells on $\mathrm{Ta}$ and $\mathrm{Cr}$ surfaces, Biomaterials 27 (8) (2006) 1346-1354.

[53] M.S. Lord, C. Modin, M. Foss, M. Duch, A. Simmons, F.S. Pedersen, B.K. Milthorpe, F. Besenbacher, Monitoring cell adhesion on tantalum and oxidised polystyrene using a quartz crystal microbalance with dissipation, Biomaterials 27 (26) (2006) 4529-4537.

[54] A. Stank, D.B. Kokh, J.C. Fuller, R.C. Wade, Protein binding pocket dynamics, Acc. Chem. Res. 49 (5) (2016) 809-815.

[55] A. Teo, S. Dimartino, S.J. Lee, K.K.T. Goh, J. Wen, I. Oey, S. Ko, H.-S. Kwak, Interfacial structures of whey protein isolate (WPI) and lactoferrin on hydrophobic surfaces in a model system monitored by quartz crystal microbalance with dissipation (QCM-D) and their formation on nanoemulsions, Food Hydrocolloids 56 (2016) 150-160.

[56] M. Rodahl, B. Kasemo, Frequency and dissipation-factor responses to localized liquid deposits on a QCM electrode, Sens. Actuat. B - Chem. 37 (1-2) (1996) $111-116$.

[57] R.H. Walters, K.H. Jacobson, J.A. Pedersen, R.M. Murphy, Elongation kinetics of polyglutamine peptide fibrils: a quartz crystal microbalance with dissipation study, J. Mol. Biol. 421 (2-3) (2012) 329-347.

[58] M.C. Vyner, L.N. Liu, H.D. Sheardown, B.G. Amsden, The effect of elastomer chain flexibility on protein adsorption, Biomaterials 34 (37) (2013) $9287-$ 9294.

[59] P. Jain, A. Soshee, S.S. Narayanan, J. Sharma, C. Girard, E. Dujardin, C. Nizak Selection of arginine-rich anti-gold antibodies engineered for plasmonic colloid self-assembly, J. Phys. Chem. C 118 (26) (2014) 14502-14510.

[60] P.X. Jia, M. He, Y.K. Gong, X. Chu, J.F. Yang, J. Zhao, Probing the adjustments of macromolecules during their surface adsorption, ACS Appl. Mater. Int. 7 (12) (2015) 6422-6429.

[61] L.L. del Mercato, P.P. Pompa, G. Maruccio, A. Della Torre, S. Sabella, A.M. Tamburro, R. Cingolani, R. Rinaldi, Charge transport and intrinsic fluorescence in amyloid-like fibrils, PNAS 104 (46) (2007) 18019-18024.

[62] A. Rizzo, N. Solin, L.J. Lindgren, M.R. Andersson, O. Inganas, White light with phosphorescent protein fibrils in OLEDs, Nano Lett. 10 (6) (2010) 2225 2230. 